\title{
Game Theoretical Mechanism Design Methods
}

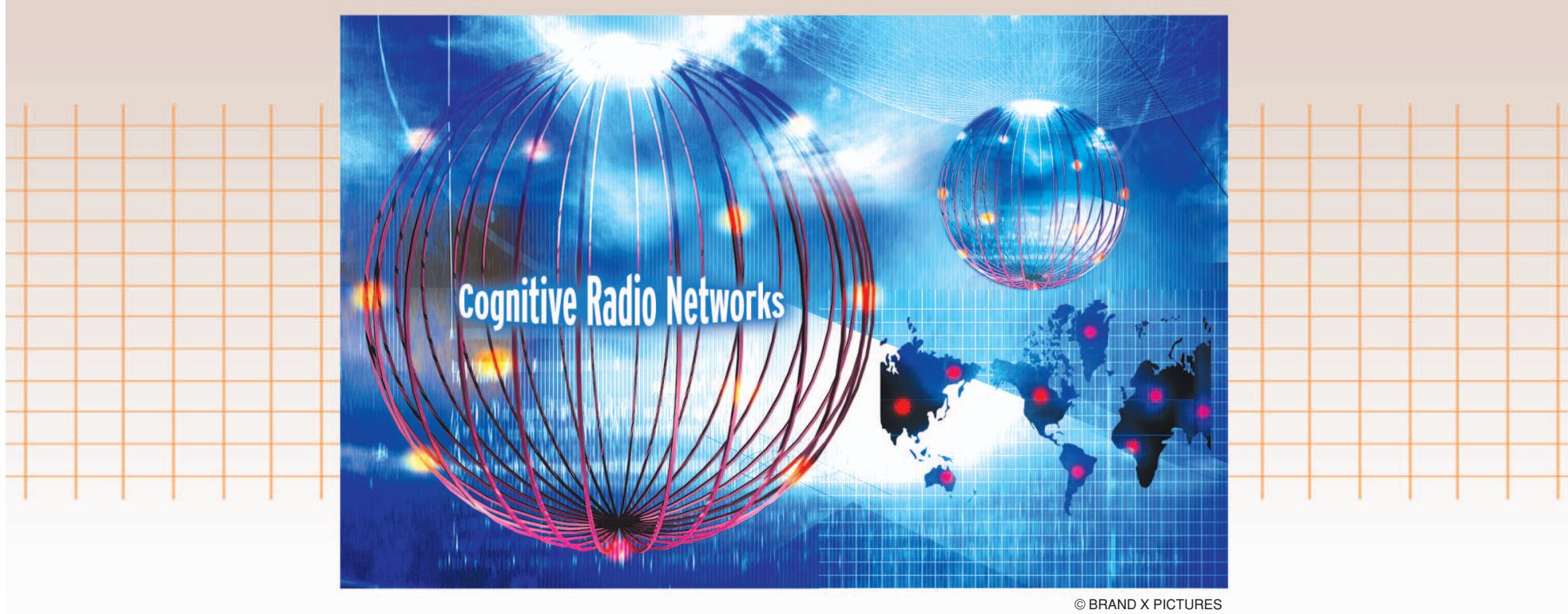

\section{Suppressing cheating in cognitive radio networks}

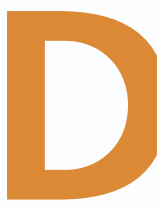

ynamic spectrum access with cognitive radios has become a promising approach to improve spectrum efficiency by adaptively coordinating different users' access according to spectrum dynamics. However, selfish users competing with each other for spectrum may exchange false private information or collude with others to get more access to the spectrum and achieve higher profits. In this article, we investigate two game-theoretical mechanism design methods to suppress cheating and collusion behavior of selfish users: a self-enforcing truth-telling mechanism for unlicensed spectrum sharing and a collusionresistant multistage dynamic spectrum pricing game for licensed spectrum sharing.

\section{INTRODUCTION}

With the emergence of new wireless applications and devices, the last decade has witnessed a dramatic increase in the demand for radio spectrum, which has forced government regulatory bodies, such as the U.S. Federal Communications Commission (FCC), to review their policies. Since the bandwidth demands may vary rapidly along the time and space dimensions, the traditional rigid allocation policies by the FCC have severely hindered efficient utilization of scarce spectrum. Hence, dynamic spectrum access, with the aid of cognitive radio technology [1], has become a promising approach, enabling wireless devices to utilize the spectrum adaptively and efficiently. 
Cognitive radio, featured with cognitive capability and reconfigurability [2], [3], enables the wireless devices not only to rapidly sense the information from the radio environment but also to dynamically adapt operational parameters, so that more efficient and intensive spectrum utilization is possible. Since 2002 , the FCC has been considering more flexible and comprehensive use of spectrum resources [4].

Researchers have also proposed various approaches to optimally share the available resources using cognitive radio technology in different scenarios. Since competitors for spectrum rights often belong to different authorities, they have no incentive to cooperate with each other and may act selfishly to maximize their own revenues. Therefore, game theory, which analyzes the conflict and cooperation among intelligent, rational decision makers, is an excellent tool and has been widely used in designing efficient spectrum sharing schemes.

In [5] and [6], the authors investigated whether spectrum efficiency and fairness can be obtained by modeling the spectrum sharing as a repeated game. The authors in [7] proposed local bargaining to achieve distributed conflict-free spectrum assignment that adapted to network topology changes. In [8], a no-regret learning algorithm using the correlated equilibrium concept to coordinate the secondary spectrum access was considered. Various auction and pricing approaches were proposed for efficient spectrum allocation, such as auction games for interference management [9], [10], the demand responsive pricing framework [11], and pricing for bandwidth sharing between WiMAX networks and WiFi hot spots [12]. A beliefassisted distributive double auction was proposed in [13] that maximized both primary and secondary users' revenues, and a game-theoretical overview for dynamic spectrum sharing was presented in [14]. [A primary user (or licensed user) refers to a spectrum license holder, e.g., TV transmitter, radar transmitter; a secondary user (or unlicensed user) refers to a user who has no spectrum license, e.g., users in the industrial, scientific and medical (ISM) band.]

Although the approaches listed above have boosted the spectrum efficiency, most of them are based on the assumption that the players (e.g., wireless users/devices) are honest and will not cheat. Nevertheless, selfish players aim only to maximize their own interests; if they believe their interests can be further increased by cheating, the users will no longer behave honestly, which usually results in a disastrous outcome for the spectrum sharing game. Therefore, designing a robust spectrum sharing scheme that can suppress cheating behaviors of selfish users is of critical importance.

Motivated by the preceding, mechanism design theory [15], whose founders, L. Hurwicz, E.S. Maskin, and R.B. Myerson, won the Nobel Prize in Economics in 2007, is a powerful tool to implement an optimal system-wide solution to a decentralized optimization problem with self-interested players. By carefully setting up the structure of the game, each player has an incentive to behave as the system designer intends, which results in a desired outcome. In this article, we investigate mechanism design-based dynamic spectrum access approaches in two scenarios: spectrum sharing in unlicensed bands and licensed bands. First, a self-enforcing truth-telling mechanism for unlicensed spectrum sharing is proposed based on repeated game modeling, in which the selfish users are motivated to share unlicensed spectrum, under the threat of punishment, and their cheating behavior is suppressed with the aid of a transfer function. The transfer function represents the payment that a user receives (or makes if it is negative) based on the private information he/she announces in the spectrum sharing game. In the proposed mechanism, it is shown that the users can get the highest utility only by announcing their true private information. Then, a collusion-resistant multistage dynamic spectrum pricing game for licensed spectrum sharing is proposed to optimize the overall spectrum efficiency and combat possible user collusion. Both approaches are demonstrated to alleviate the degradation of the system performance due to selfish users cheating.

\section{GAME THEORY BASICS FOR COGNITIVE RADIO NETWORKS}

In cognitive radio networks, the network users make intelligent decisions on spectrum usage and communication parameters based on the sensed spectrum dynamics and other users' decisions. Furthermore, the network users who compete for spectrum resources may have no incentive to cooperate with each other and behave selfishly. Therefore, it is natural to study the intelligent behaviors and interactions of selfish network users from the game theoretical perspective.

Game theory is a mathematical tool that analyzes the strategic interactions among multiple decision makers. Three major components in a strategic-form game model are the set of players, the strategies/action space of each player, and the utility/payoff function, which measures the outcome of the game for each player. In cognitive radio networks, the competition and cooperation among the cognitive network users can be well modeled as a spectrum sharing game. Specifically, in open spectrum sharing, the players are all the secondary users that compete for unlicensed spectrum; in licensed spectrum sharing, where primary users lease their unused bands to secondary users, the players include both the primary and secondary users.

The strategy space for each player may vary according to the specific spectrum sharing scenario. For instance, the strategy space of secondary users in open spectrum sharing may include the transmission parameters they want to adopt, such as the transmission powers, access rates, time duration, etc.; while in licensed spectrum trading, their strategy space includes which licensed bands they want to rent and how much they would pay 
for leasing those licensed bands. For the primary users, the strategy space may include which secondary users they would lease each of their unused bands to and how much they will charge for each band. The utility functions for different users are accordingly defined to characterize various performance criteria. In open spectrum sharing, the utility function for the secondary users is often defined as a nondecreasing function of the quality of service (QoS) they receive by utilizing the unlicensed band; in licensed spectrum trading, the utility function for the users often represents the monetary gains (e.g., revenue minus cost) by leasing the licensed bands.

In a noncooperative spectrum sharing game with selfish network users, each user only aims to maximize his/her own utility by choosing an optimal strategy. And the outcome of the noncooperative game is often measured by the Nash equilibrium (NE). The NE is defined as the set of strategies for all the users such that no user can improve his/her utility by unilaterally deviating from the equi-

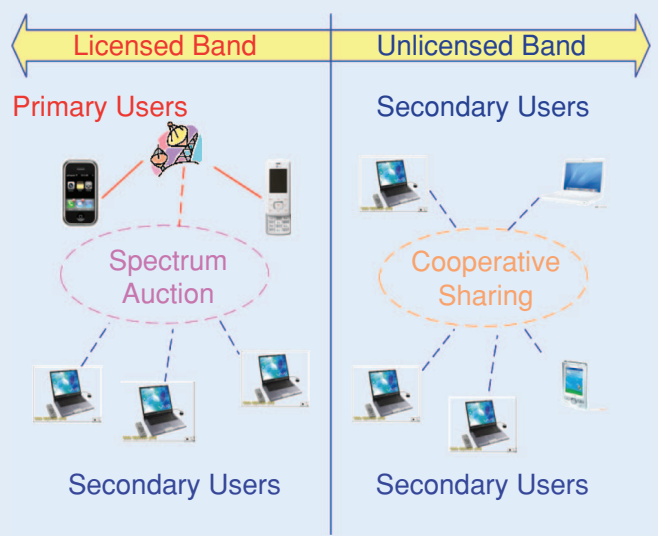

(a)

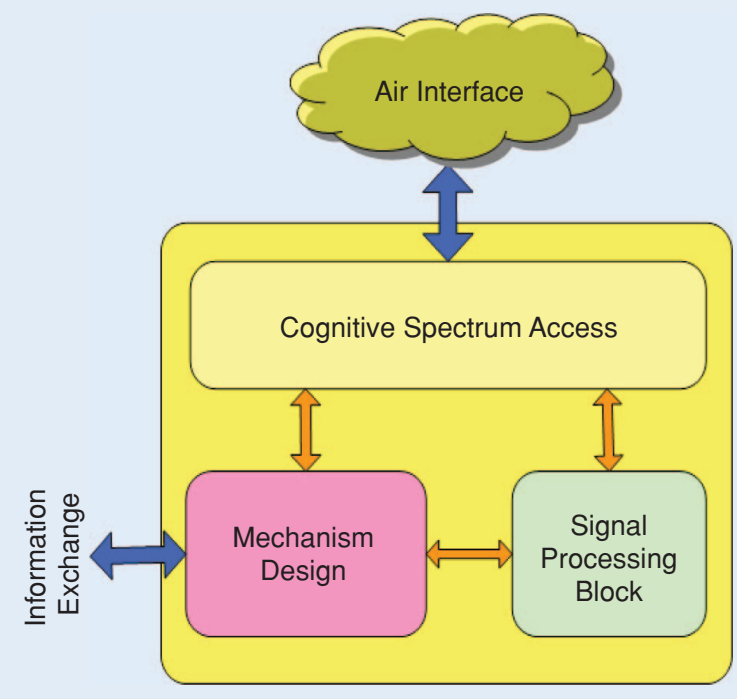

(b)

[FIG1] System model and cognitive radio architecture. (a) Spectrum sharing in licensed/unlicensed bands. (b) Cognitive radio architecture. librium strategy, given that the other users adopt the equilibrium strategies. So the NE indicates that no individual user would have the incentive to choose a different strategy. However, in a static noncooperative game, that is, the game is played only once, the users are myopic and only care about the current utility, and the competition between selfish users often results in an NE that is not system efficient. Therefore, stimulation of cooperation among selfish users is very important to achieve social welfare. Considering that the spectrum sharing in cognitive radio networks is a dynamic process, repeated game models better capture interactions in long-run scenarios. In repeated game modeling, the users play a similar static game many times, so they will make decisions conditioned on other users' past moves. In this way, cooperation can be enforced by establishing the threat of punishment, individual reputation, mutual trust, and so on.

Due to the selfish nature of the network users, they will not reveal their true private information (e.g., channel quality values in open spectrum sharing and the evaluation of licensed bands in licensed spectrum trading) if they believe that cheating can further improve their own utility values. Cheating usually results in a disastrous outcome. Therefore, certain incentives have to be provided so as to suppress users' cheating behaviors. To this end, mechanism design [15] can be employed to implement an optimal system-wide solution to a decentralized optimization problem even though the players are self-interested. By carefully designing the rules of the game, selfish users in the spectrum sharing game will behave as the system designer intends, resulting in a desired outcome with social welfare.

\section{SPECTRUM SHARING IN UNLICENSED BANDS}

Consider the spectrum sharing in unlicensed bands shown in Figure 1(a), where $K$ secondary users coexisting in the same area compete for spectrum access rights in an open, unlicensed band. The cognitive radio architecture is shown in Figure 1(b), which can interface with the radio environment to gather and exchange channel measurements among distributed users, analyze the collected measurements via signal processing blocks, and assign frequency bands to specific users by mechanism design to optimize the spectrum allocation efficiency.

\section{ONE-SHOT UNLICENSED SPECTRUM SHARING GAME}

As mentioned in the previous section, in the unlicensed spectrum sharing the players are all the secondary users, and the strategy for user $i$ is the set of transmission power level $p_{i}$, with $p_{i} \in\left[0, p_{i}^{\max }\right]$ and $p_{i}^{\max }$ representing the peak power constraint. The utility function for user $i$ can be defined as an increasing function of his/her QoS, and we use the data throughput as the utility for simplicity, which is expressed as

$$
R_{i}\left(p_{1}, p_{2}, \ldots, p_{K}\right)=\log _{2}\left(1+\frac{p_{i}\left|h_{i i}\right|^{2}}{N_{0}+\sum_{j \neq i} p_{j}\left|h_{j i}\right|^{2}}\right) .
$$

In (1), $\left|h_{j i}\right|$ represents the channel gain from user $j$ 's transmitter to user $i$ 's receiver, $N_{0}$ is the noise power, and the mutual interference is treated as Gaussian noise. 
It is shown that the only NE for this static spectrumsharing game is $\left(p_{1}^{\max }, p_{2}^{\max }, \ldots, p_{K}^{\max }\right)$. In the $\mathrm{NE}$, the unlicensed spectrum is excessively exploited as all the selfish users occupy the spectrum with maximal transmission power. Thus, each of them will receive a very low payoff due to the strong mutual interference. As the spectrum sharing lasts over quite a long period of time, a punishment-based repeated game model is proposed in order to provide users with the incentive to cooperate.

\section{REPEATED GAME WITH COOPERATION STIMULATION}

In a repeated game, the overall payoff is represented as a normalized discounted summation of the payoff at each stage game, i.e., [16], [17],

$$
U_{i}=(1-\delta) \sum_{n=0}^{+\infty} \delta^{n} R_{i}[n]
$$

where $R_{i}[n]$ is user $i$ 's payoff at the $n$th stage, $\delta(0<\delta<1)$ is the discount factor which indicates that a user values the current stage payoff more than the payoffs in future stages, and $(1-\delta)$ can be viewed as a normalization factor. As $R_{i}[n]$ is assumed to be a finite value, $U_{i}$ is well defined in the repeated game. If $\delta$ is close to one, we say that the user is patient; if $\delta$ is close to zero, we say that the user is myopic. In general, the spectrum sharing in unlicensed bands lasts for a long time, and we can assume that $\delta$ is close to one. Because the users care about not only the current payoff but also the rewards in the future, they have to constrain their behavior in the present to keep a good credit history; otherwise, a bad reputation may cost even more in the future.

The optimal strategy for the selfish users in the one-shot game is to transmit with the maximal power, which leads to a very low payoff $r_{i}^{S}$ (the superscript $\mathrm{S}$ stands for selfish). However, if all the players follow some predetermined rules to share the spectrum, higher expected one-shot payoffs $r_{i}^{C}(C$ stands for cooperation) may be achieved, i.e., $r_{i}^{C}>r_{i}^{S}$ for $i=1,2, \ldots, K$. For example, the cooperation rule may require that only several players access the spectrum simultaneously, and hence mutual interference is greatly reduced. Nevertheless, without any commitment, selfish players always want to deviate from cooperation. One player can take advantage of the others by transmitting in time slots that he/she is not supposed to and consequently gets a larger instantaneous payoff $r_{i}^{D}$ ( $D$ stands for deviation).

Although cooperation is not a stable equilibrium in the one-shot game, it can be enforced by the threat of punishment in the repeated game. The aim of punishment is to prevent deviating behaviors from happening. As long as the punishment is long enough to negate the reward from a one-time deviation, no player has the incentive to deviate. The strategy, called punish-and-forgive, is stated as follows. The game starts from the cooperative stage at time 0 and will stay in the cooperative stage until some deviation happens at an arbitrary time slot $T_{0}$. Then, the game jumps into the punishment stage for the next $(T-1)$ time slots before the misbehavior is forgiven and cooperation resumes from the $\left(T_{0}+T\right)$ th time slot. In the cooperative stage, every player shares the spectrum in a cooperative way according to their agreement; while in the punishment stage, players occupy the spectrum noncooperatively as they would do in the one-shot game. By the Folk Theorem with Nash threats [16], provided $r_{i}^{C}>r_{i}^{S}$ for all $i=1,2, \ldots, K$, there exists $\bar{\delta}<1$, such that for sufficiently large discount factor $\delta>\bar{\delta}$, the game has a subgame perfect equilibrium with discounted utility $r_{i}^{C}$, if all players adopt the punish-and-forgive strategy.

The duration of punishment, $T$, can be determined by analyzing the incentive of the players. Assume that user $i$, who deviates at time $T_{0}$, will have his/her instantaneous payoff at that slot increased to at most $\overline{R_{i}^{D}}$. After $T_{0}$, the punishment stage will last for the next $T-1$ slots. Denote the overall payoff for user $i$ in the repeated game by $U_{i}^{D}$ if he/she deviates, then the expected value $u_{i}^{D}$ is upper-bounded by

$$
\begin{aligned}
u_{i}^{D} \triangleq E_{h_{j i}}\left[U_{i}^{D}\right] \leq(1-\delta) & \\
& \cdot\left(\sum_{n=0}^{T_{0}-1} \delta^{n} r_{i}^{C}+\delta^{T_{0}} \overline{R_{i}^{D}}+\sum_{n=T_{0}+1}^{T_{0}+T-1} \delta^{n} r_{i}^{S}+\sum_{n=T_{0}+T}^{+\infty} \delta^{n} r_{i}^{C}\right),
\end{aligned}
$$

where the expectation of $U_{i}^{D}$ is with respect to all channel realizations $\left\{h_{j i}\right\}$ s. In general, cooperation guarantees an average payoff $r_{i}^{C}$ at each time slot, but the worst-case instantaneous payoff at time $T_{0}$ would be zero, which means user $i$ has no right to utilize the open spectrum at that time. Denote the overall payoff for user $i$ without deviation by $U_{i}^{C}$, then its expected value $u_{i}^{C}$ is lower bounded by

$$
u_{i}^{C} \triangleq E_{h_{j i}}\left[U_{i}^{C}\right] \geq(1-\delta)\left(\sum_{n=0}^{T_{0}-1} \delta^{n} r_{i}^{C}+0+\sum_{n=T_{0}+1}^{+\infty} \delta^{n} r_{i}^{C}\right)
$$

From the selfish user's perspective, the strategy with the higher payoff is the better choice, so $T$ should be chosen such that $u_{i}^{C}>u_{i}^{D}$ for all $i=1,2, \ldots, K$ to prevent the users from deviating.

\section{BAYESIAN MECHANISM DESIGN FOR TRUTH-TELLING}

In the spectrum sharing game, users can exchange their channel state information over a common control channel. Based on this information, each individual user can independently determine who is eligible to transmit in the current time slot according to certain spectrum sharing rules. To utilize the unlicensed spectrum efficiently, only a few users with small mutual interference can access the spectrum simultaneously. In an urban area with high user density, it is proper to allow only one user to occupy the spectrum in each time slot [22]. Then an efficient spectrum sharing rule can be defined to assign the spectrum rights to the user with the highest 
instantaneous received signal power, i.e., the cooperation rule is $d\left(g_{1}, g_{2}, \ldots, g_{K}\right)=\operatorname{argmax}_{i} g_{i}$, with $g_{i}=p_{i}^{\max }\left|h_{i i}\right|^{2}$. The user with index $d\left(g_{1}, g_{2}, \ldots, g_{K}\right)$ can access the channel, with data throughput defined as $R_{i}\left(g_{i}, d\left(g_{1}, g_{2}, \ldots, g_{K}\right)\right)$ $\triangleq \log _{2}\left(1+\left(g_{i} / N_{0}\right)\right)$, if $d\left(g_{1}, g_{2}, \ldots, g_{K}\right)=i$, and 0 otherwise. The private information $\left(g_{1}, g_{2}, \ldots, g_{K}\right)$ needs to be exchanged among the users. Nevertheless, this spectrumsharing rule favors the user who claims the highest received signal power, so the selfish users tend to exaggerate their claimed $g_{i}$ value to acquire more spectrum access. Therefore, the users have to be motivated to report their true private information $g_{i}$ s to guarantee efficient spectrum usage, and Bayesian mechanism design [16] is employed to provide users with the incentive to tell the truth.

To be specific, the user claiming a higher $g_{i}$ value is asked to pay a tax, and the amount of tax will increase as the claimed value increases, whereas the users

\section{COGNITIVE RADIO ENABLES THE WIRELESS DEVICES NOT ONLY TO RAPIDLY SENSE THE INFORMATION FROM THE RADIO ENVIRONMENT BUT ALSO TO DYNAMICALLY ADAPT OPERATIONAL PARAMETERS.}

represents the expected sum of all the other users' data throughput according to the aforementioned spectrum sharing rule $d\left(g_{1}, g_{2}, \ldots, g_{K}\right)$, given that user $i$ claims $\hat{g}_{i}$. Intuitively, if user $i$ claims a higher $\hat{g}_{i}$, he/she will gain a greater chance to access the spectrum, and all the other users will have a smaller spectrum share. So $\Phi_{i}\left(\hat{g}_{i}\right)$ will decrease, $\sum_{j=1, j \neq i}^{K} \Phi_{j}\left(\hat{g}_{j}\right) /(K-1) ; \quad$ will increase, and therefore, the transfer value for user $i$ tends to decrease. This may negate the additional gain from more spectrum access through exaggerating the channel gain. On the contrary, if the claimed $\hat{g}_{i}$ is lower, user $i$ will receive some compensation at the cost of less chance to occupy the spectrum. Therefore, in the proposed mechanism, it is equilibrium that each user reports his/her true private information. We can also show that all users' transfer values add up to zero at any time. This means that the monetary transfer is exchanged with neither surplus nor deficit, and therefore the proposed mechanism is suitable for the unlicensed spectrum sharing.

\section{PERFORMANCE EVALUATION}

We assume a homogeneous spectrum sharing scenario, in which all the secondary users have the same maximal transmission power, the channel gains $\left\{h_{i i}\right\} \sim \mathcal{C N}(0,1)$, and $\left\{h_{i j}\right\} \sim \mathcal{C N}(0,1)$. In Figure 2, we illustrate the idea of the punishment-based repeated game with two users. Assume user 1 deviates from cooperation at time 150, and the duration of the punishment stage is $T=150$. According to the punish-and-forgive strategy, the game will stay in the punishment stage from time slot 151 to 300 . We simulated 100 independent runs, in each of which a series of i.i.d. channel realizations is generated and the instantaneous payoff at each slot is calculated. Figure 2 shows the averaged payoff for the deviating user. We can see that although the player gets a high payoff at time slot 150 by deviation, the temporary profit will be negated in the punishment stage. Hence, considering the consequence of deviation, the selfish users have no incentive to deviate. Then we examine the proposed cheat-proof strategy in a three-user spectrum sharing game. At one specific time slot, the true channel gains are assumed to be $\tilde{g}_{1}=0.4, \tilde{g}_{2}=0.8$, and $\tilde{g}_{3}=1.1$.In Figure 3 , the expected overall payoff (throughput plus transfer) versus the claimed channel gains is shown for each user, given the other two are honest. From the figure we see that the overall expected

[FIG2] Illustration of the punishment-based repeated game. 
payoff is maximized only if the player honestly claims its true information. Therefore, the users are self-enforced to tell the truth with the proposed mechanism.

\section{SPECTRUM SHARING IN LICENSED BANDS}

Consider a wireless network where multiple primary users and secondary users operate simultaneously, as shown in Figure 4. The network users are equipped with cognitive radio devices, which enables a more flexible spectrum access by allowing secondary users to gain access to multiple primary operators or having multiple secondary users compete for available spectrum. A spectrum pooling [20] architecture is used to collect unused or under-used licensed spectra and divide them into orthogonal frequency channels based on orthogonal frequencydivision multiplexing (OFDM) techniques. There may not be a centralized authority. A management point may exist to handle the billing information for spectrum leasing activities, and control channels are assumed for exchanging spectrum sharing information. The characteristics of spectrum resources may vary over frequency, time, and space due to user mobility, channel variations, or wireless traffic fluctuations.

\section{DOUBLE AUCTION MECHANISM FOR DYNAMIC SPECTRUM ALLOCATION}

In general, the primary users have to pay certain operating costs to acquire the spectrum licenses while the authorized spectrum of the primary users may not be fully utilized over time, so they prefer to lease the unused channels to secondary users for monetary gains. The utility function for a primary user $P_{i}$ can be defined as the total payments collected from all the secondary users who lease certain channels from $P_{i}$ minus the acquisition cost of $P_{i}$ for the licensed channels. On the other hand, as the unlicensed spectrum becomes more and more crowded, the secondary users may try to lease some unused channels from the primary users for more communication gains by providing leasing payments. Then, the utility function for a secondary user $S_{j}$ can be defined as the entire reward (gains from communication) if $S_{j}$ successfully leases some licensed channels minus the charge to $S_{j}$.

We assume that all users are selfish, rational, and not malicious, which means that their objectives are to maximize their own utilities and not to cause dam- age to other users. Then, the users have conflicting interests with each other: the primary users want to earn as much revenue as possible by leasing the unused channels, while the secondary users aim to obtain more spectrum usage rights by providing the least possible payments to the primary users. Furthermore, spectrum allocation involves multiple channels over time. Therefore, the interaction between primary and secondary users can be modeled as a multistage noncooperative pricing game [16], [17]. However, selfish users will not reveal their private information to the others in general. This leads to a noncooperative game with incomplete information, which is complex and difficult to study as the users do not know the perfect strategy profile of others. Therefore, proper mechanisms have to be applied to guarantee that it is not harmful for the selfish users to disclose the private information. Based on our

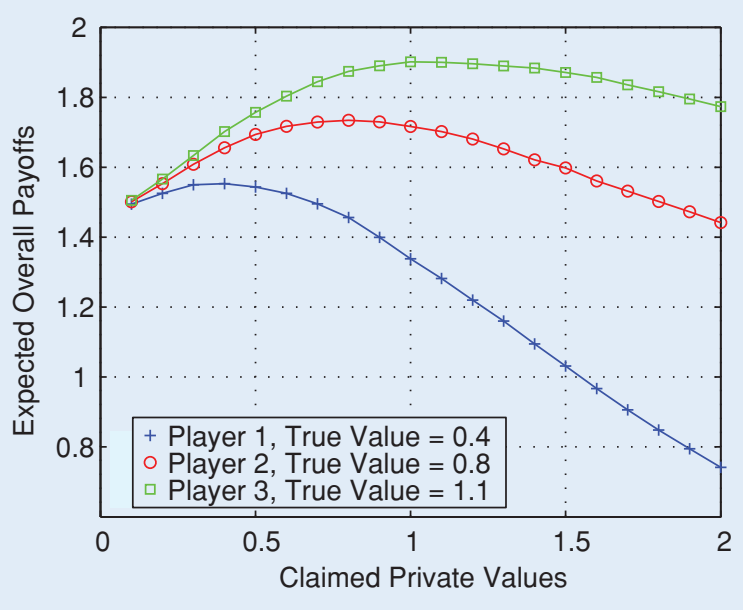

[FIG3] The expected overall payoff versus different claimed values.

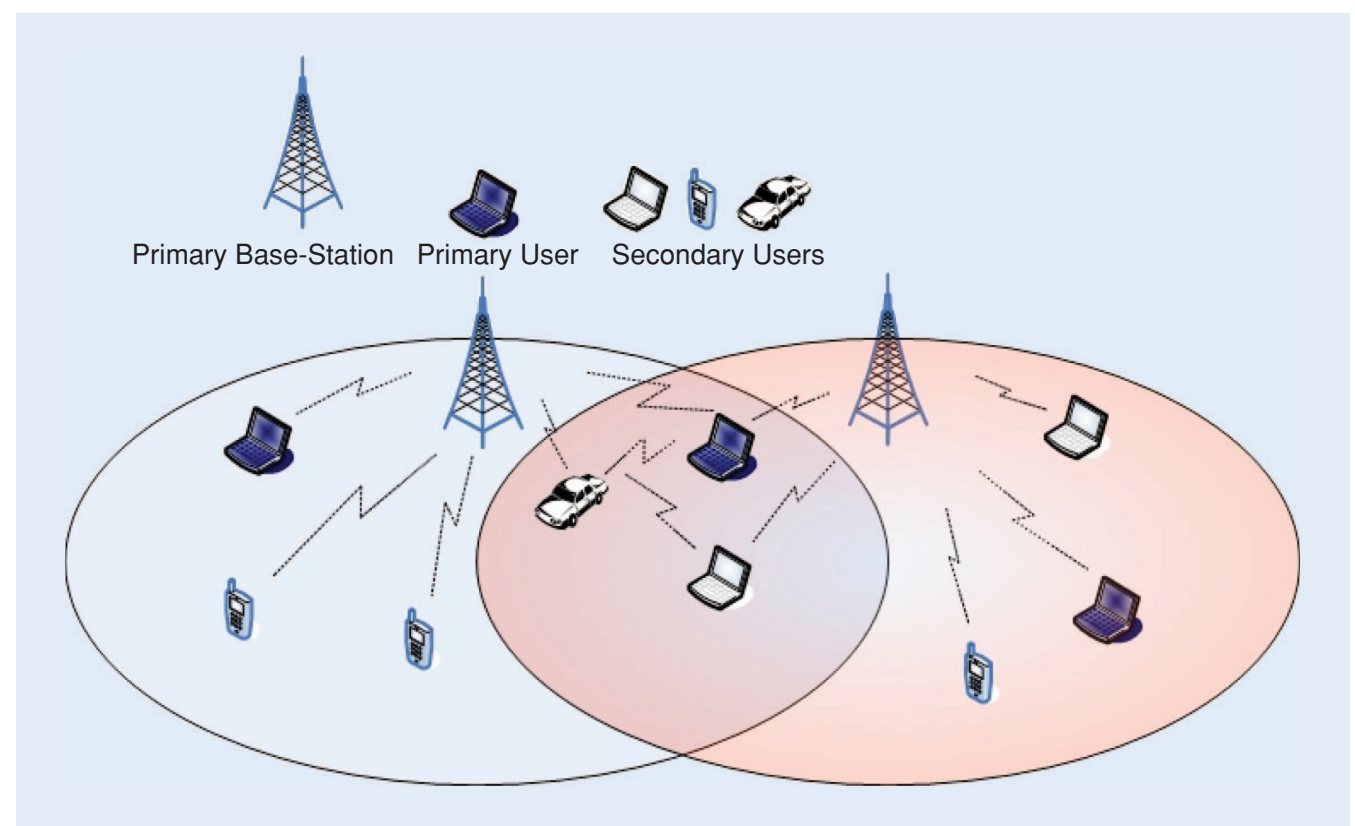

[FIG4] Illustration of dynamic spectrum access networks. 
game setting, the auction mechanism [18] can be employed to formulate and analyze this spectrum pricing game.

In the spectrum auction, the primary users are viewed as the auctioneers, who determine the resource allocation and the prices based on bids from the secondary users; on the other hand, the secondary users compete with each other to buy the permission of using the primary users' channels. Note that multiple primary and secondary users coexist, so not only the secondary users but also the primary users need to compete with each other by offering attractive prices on their licensed bands. This indicates the double auction scenario [18], where multiple buyers bid to buy goods from multiple sellers and the sellers also compete with each other simultaneously. In the spectrum double auction, the primary/secondary users express their charge/payment of a certain licensed band in the form of an ask or a bid, to make beneficial transactions. The double auction mechanism is in general highly efficient, such as in the New York Stock Exchange, and incentive compatibility can be assured, which indicates that no selfish user will cheat on the auction mechanism unilaterally.

\section{COLLUSION-RESISTANT}

\section{STRATEGY WITH BELIEF ESTABLISHMENT}

Users may cheat whenever they believe cheating behaviors can help increase their payoffs. One prevalent cheating behavior, the bidding collusion among users, has been generally overlooked. The collusive cheating behaviors among several selfish users will pose severe threats to efficient spectrum allocation and

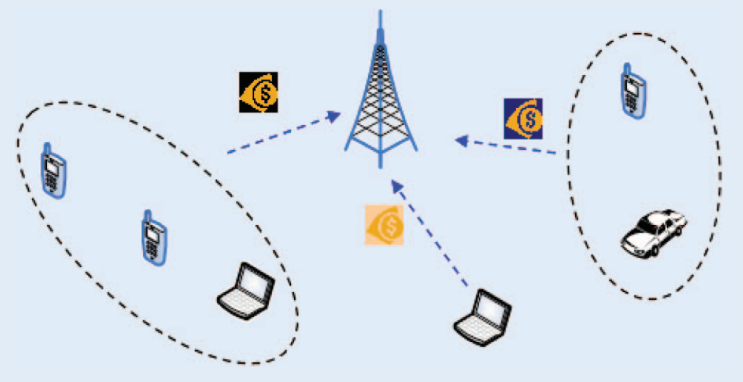

[FIG5] User collusion in pricing-based dynamic spectrum allocation.

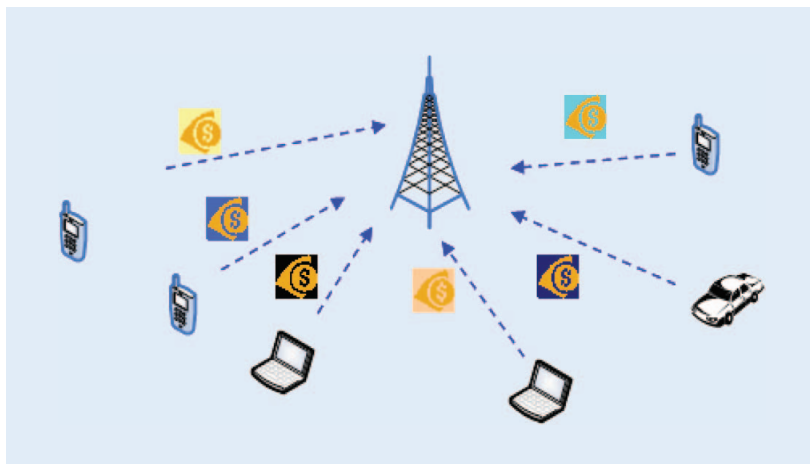

[FIG6] No collusion in pricing-based dynamic spectrum allocation. deteriorate the efficiency of the game outcomes. To be specific, the bidders (or sellers) act collusively and engage in bid rigging with a view to obtaining lower prices (or higher prices). The resulting arrangement is called a bidding ring. In the scenarios of auction-based spectrum allocation, a bidding ring among primary users (or secondary users) will result in increasing their utilities by collusively leasing the spectrum channels at a higher price (or at a lower price).

In Figures 5 and 6, we illustrate a snapshot of pricing-based dynamic spectrum access networks with and without user collusion, respectively. In these figures, we consider the primary base station as the primary user and the unlicensed mobile users as secondary users. When there is no user collusion as in Figure 6, the pricing interactions between the primary user and secondary users lead to efficient spectrum allocation. When there exist several bidding rings as in Figure 5, each bidding ring will elicit only one effective bid for spectrum resources, which distorts the supply and demand of spectrum resources and yields inefficient spectrum allocation. Further, in the extreme case that all secondary users collude with each other, arbitrarily low bid price will become eligible. Thus, collusionresistant dynamic spectrum allocation is important for efficient next-generation wireless networking.

In a traditional ascending-price open auction [18], where there is one seller and multiple buyers (or one buyer and multiple sellers), to combat collusion, the seller/buyer can enhance his/her payoff by setting an optimal reserve price, which means the seller/buyer will not sell/buy the spectrum resources at prices lower/higher than the reserve price. A similar idea can also be applied to the spectrum auction game with multiple primary and secondary users.

First, let's consider an example network with multiple secondary users and one primary (MSOP) user $P_{i}$ as shown in Figure 6 . The standard open ascending price auction is chosen for the secondary users to compete for the spectrum resources, which is theoretically equivalent to sealed-bid, second-price auction [18]. Denote the highest and second highest reward value among all effective secondary users as $v_{(1)}$ and $v_{(2)}$, respectively. Let's denote the optimal reserve price for primary user $P_{i}$ by $\phi_{r, p_{i}}$. Then, the spectrum channel can be leased by $P_{i}$ if and only if $v_{(1)}>\phi_{r, p_{i}}$. Moreover, if $v_{(2)}>\phi_{r, p_{i}}$, the spectrum channel is leased for $v_{(2)}$; otherwise, it is leased at the reserve price $\phi_{r, p_{i}}$.

Let $F_{v_{(1)}}(x)$ and $F_{v_{(2)}}(x)$ denote the cumulative distribution functions (CDFs) of $v_{(1)}$ and $v_{(2)}$, respectively. Let $f_{v_{(1)}}(x)$ and $f_{v_{(2)}}(x)$ denote the probability density functions (PDFs) of $v_{(1)}$ and $v_{(2)}$, respectively. Thus, the expected utility gain of the primary user with reserve price $\phi_{r, p_{i}}$ by leasing her/his $j$ th channel can be written as

$$
\begin{aligned}
E\left[U_{p_{i}}^{j}\left(\phi_{r, p_{i}}\right)\right]= & \left(\phi_{r, p_{i}}-c_{i}^{j}\right)\left(F_{v_{(2)}}\left(\phi_{r, p_{i}}\right)-F_{v_{(1)}}\left(\phi_{r, p_{i}}\right)\right) \\
& +\int_{\phi_{r, p_{i}}}^{M}\left(z-c_{i}^{j}\right) f_{v_{(2)}}(z) d z,
\end{aligned}
$$

where $M$ represents the largest possible reward value of primary user $P_{i}$ 's $j$ th channel among the competing secondary 
users, and $c_{i}^{j}$ denotes the channel acquisition cost of primary user $P_{i}$. Note that the first term on the right-hand side (RHS) of (7) represents the utility when the spectrum channel is leased at the reserve price. This happens if $v_{(1)}>\phi_{r, p_{i}}$ but $v_{(2)}<\phi_{r, p_{i}}$ because this channel won't be able to be leased at the second highest bid but the reserve price. Since event $\left\{v_{(2)}<\phi_{r, p_{i}}\right\}=$ $\left\{v_{(2)}<\phi_{r, p_{i}}<v_{(1)}\right\} \bigcup\left\{v_{(1)} \leq \phi_{r, p_{i}}\right\}$, where the latter two events are mutually exclusive, we know that $\left(F_{v_{(2)}}\left(\phi_{r, p_{i}}\right)-F_{v_{(1)}}\left(\phi_{r, p_{i}}\right)\right)$ is the probability that event $\left\{v_{(2)}<\phi_{r, p_{i}}<v_{(1)}\right\}$ happens. The second term on the RHS of (7) represents the expected utility when $v_{(2)} \geq \phi_{r, p_{i}}$.

Assuming that an interior maximum exists for (7), the optimal reserve price $\phi_{r, p_{i}}^{*}$ satisfies the following first-order condition of $(7)$,

$$
F_{v_{(2)}}\left(\phi_{r, p_{i}}^{*}\right)-F_{v_{(1)}}\left(\phi_{r, p_{i}}^{*}\right)-\left(\phi_{r, p_{i}}^{*}-c_{i}^{j}\right) f_{v_{(1)}}\left(\phi_{r, p_{i}}^{*}\right)=0 .
$$

Thus the optimal reserve price can be determined by (8) if the statistical descriptions for $v_{(1)}$ and $v_{(2)}$ are available. Similarly, in the scenario with one secondary user and multiple primary users, the optimal reserve price for the secondary user can also be obtained, if the statistical descriptions for the acquisition costs among all effective primary users are available. However, due to the network dynamics and imperfect available information, the users cannot make a credible assumption about the presence of user collusion or the number of collusive users. Therefore, they need to build up certain beliefs of other users' future possible strategies to assist their decision making.

Considering that there are multiple users with private information in the spectrum allocation game, and the bid/ask prices directly affect the outcome of the game, it is more efficient to define a belief function for each user based on the publicly observable bid/ask prices instead of generating a specific belief of every other user's private information. Thus, primary/secondary users' beliefs are defined as the ratios of their bid/ask prices being accepted at different price levels.

Let $x$ and $y$ be the ask price of the primary users and the bid price of the secondary users, respectively. At each time, the ratio of asks from primary users at $x$ that have been accepted can be written as

$$
\tilde{r}_{p}(x)=\frac{\mu_{A}(x)}{\mu(x)},
$$

where $\mu(x)$ and $\mu_{A}(x)$ are the number of asks at $x$ and the number of accepted asks at $x$, respectively. Similarly, at each time during the dynamic spectrum sharing, the ratio of bids from secondary users at $y$ that have been accepted is

$$
\tilde{r}_{s}(y)=\frac{\eta_{A}(y)}{\eta(y)}
$$

where $\eta(y)$ and $\eta_{A}(y)$ are the number of bids at $y$ and the number of accepted bids at $y$, respectively. Usually, $\tilde{r}_{p}(x)$ and $\tilde{r}_{s}(y)$ can be accurately estimated if a great number of buyers and sellers are participating in the pricing at the same time. However, in our pricing game, only a relatively small number of players are involved in the spectrum sharing at a specific time. The beliefs, namely, $\tilde{r}_{p}(x)$ and $\tilde{r}_{s}(y)$ cannot be practically obtained so that we need to further consider using the historical bid/ask information to build up empirical belief values.

Take the auction game with one primary user and multiple secondary users as an example. If a bid $\tilde{y}>y$ is rejected, the bid at $y$ will also be rejected; if a bid $\tilde{y}<y$ is accepted, the bid at $y$ will also be accepted. Generalizing the observations above to the case with multiple primary and secondary users, some new observations are as follows: if a bid $\tilde{y}>x$ is made, then an ask at $x$ will be accepted; if an ask $\tilde{x}<y$ is made, then the bid at $y$ will be accepted. According to the observations above, we can define the primary users' belief value for each potential ask at $x$ as

$$
\hat{r}_{p}(x)= \begin{cases}1 & x=0 \\ \frac{\sum_{w \geq x} \mu_{A}(w)+\sum_{w \geq x} \eta(w)}{\sum_{w \geq x} \mu_{A}(w)+\sum_{w \geq x} \eta(w)+\sum_{w \leq x} \mu_{R}(w)} & x \in(0, M), \\ 0 & x \geq M\end{cases}
$$

where $\mu_{R}(w)$ is the number of asks at $w$ that have been rejected, and $M$ is a large enough value so that asks greater than $M$ will definitely be rejected. Similarly, we can define the secondary users' belief value for each potential bid at $y$ as

$$
\hat{r}_{s}(y)= \begin{cases}0 & y=0 \\ \frac{\sum_{w \leq y} \eta_{A}(w)+\sum_{w \leq y} \mu(w)}{\sum_{w \leq y} \eta_{A}(w)+\sum_{w \leq y} \mu(w)+\sum_{w \geq y} \eta_{R}(w)} & y \in(0, M), \\ 1 & y \geq M\end{cases}
$$

where $\eta_{R}(w)$ is the number of bids at $w$ that have been rejected.

After obtaining belief values on discrete bid/ask price levels, we can use interpolation to obtain the belief function over the entire price space. Considering the characteristics of open ascending auction in the scenarios of MSOP, the secondary user with the highest reward value doesn't need to bid his/her true value to win the auction. Instead, he/she only needs to bid at the second highest possible payoff to have all the other secondary users drop out of the auction. Therefore, the secondary users' belief function (12) actually represents the CDF of $v_{(2)}$. Further, since the total number of secondary users and 
the statistics of their reward values are generally available, the CDF of $v_{(1)}$ in the scenarios of MSOP can be obtained using the order statistics [23] as

$$
F_{v_{(1)}}(x)=\prod_{i \in\{1,2, \ldots, K\}} F_{v_{i}}(x) .
$$

Therefore, the optimal reserve price $\phi_{r, p_{i}}$ for the primary user to combat user collusion in the scenarios of MSOP can be obtained from (8) using (12) and (13), and the optimal reserve price for the secondary user can be similarly obtained. Based on the above discussions, we illustrate our collusion-resistant dynamic pricing algorithm for spectrum allocation in Table 1.

\section{PERFORMANCE EVALUATION}

We consider a general scenario with multiple primary and secondary users in wireless networks as in Figure 4 and evaluate the performance of our proposed belief-assisted dynamic spectrum sharing approach. Considering a wireless network covering a $100 \mathrm{~m} \times 100 \mathrm{~m}$ area, we simulate $J$ primary users by randomly placing them in the network. Here we assume the

\section{[TABLE 1] COLLUSION-RESISTANT DYNAMIC SPECTRUM ALLOCATION.}

\section{1) INITIALIZE THE USERS' BELIEFS AND BIDS/ASKS}

- THE PRIMARY USERS INITIALIZE THEIR ASKS AS LARGE VALUES CLOSE TO M AND THEIR BELIEFS AS SMALL POSITIVE VALUES LESS THAN 1;

- THE SECONDARY USERS INITIALIZE THEIR BIDS AS SMALL VALUES CLOSE TO 0 AND THEIR BELIEFS AS SMALL POSITIVE VALUES LESS THAN 1.

2) BELIEF UPDATE BASED ON LOCAL INFORMATION

UPDATE PRIMARY AND SECONDARY USERS' BELIEFS USING (11) AND (12), RESPECTIVELY.

3) OPTIMAL RESERVE PRICE FOR PRIMARY AND SECONDARY USERS

UPDATE PRIMARY USERS' OPTIMAL RESERVE PRICES $\phi_{r . p}^{*}$ USING (8), (13) AND (11): UPDATE SECONDARY USERS' OPTIMAL RESERVE PRICES $\phi_{r, S_{i}}^{*}$ SIMILARLY.

4) OPTIMAL BIDIASK UPDATE

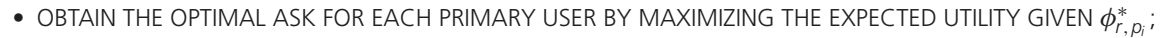

- OBTAIN THE OPTIMAL BID FOR EACH SECONDARY USER SIMILARLY GIVEN $\phi_{r, S_{i}}^{*}$

5) UPDATE LEASING AGREEMENT AND SPECTRUM POOL

IF THE CURRENT HIGHEST BID IS GREATER THAN OR EQUAL TO THE CURRENT LOWEST ASK, THE LEASING AGREEMENT WILL BE SIGNED BETWEEN THE CORRESPONDING USERS; UPDATE THE SPECTRUM POOL BY REMOVING THE ASSIGNED CHANNEL.

6) ITERATION

IF THE SPECTRUM POOL IS NOT EMPTY, GO BACK TO STEP 2.

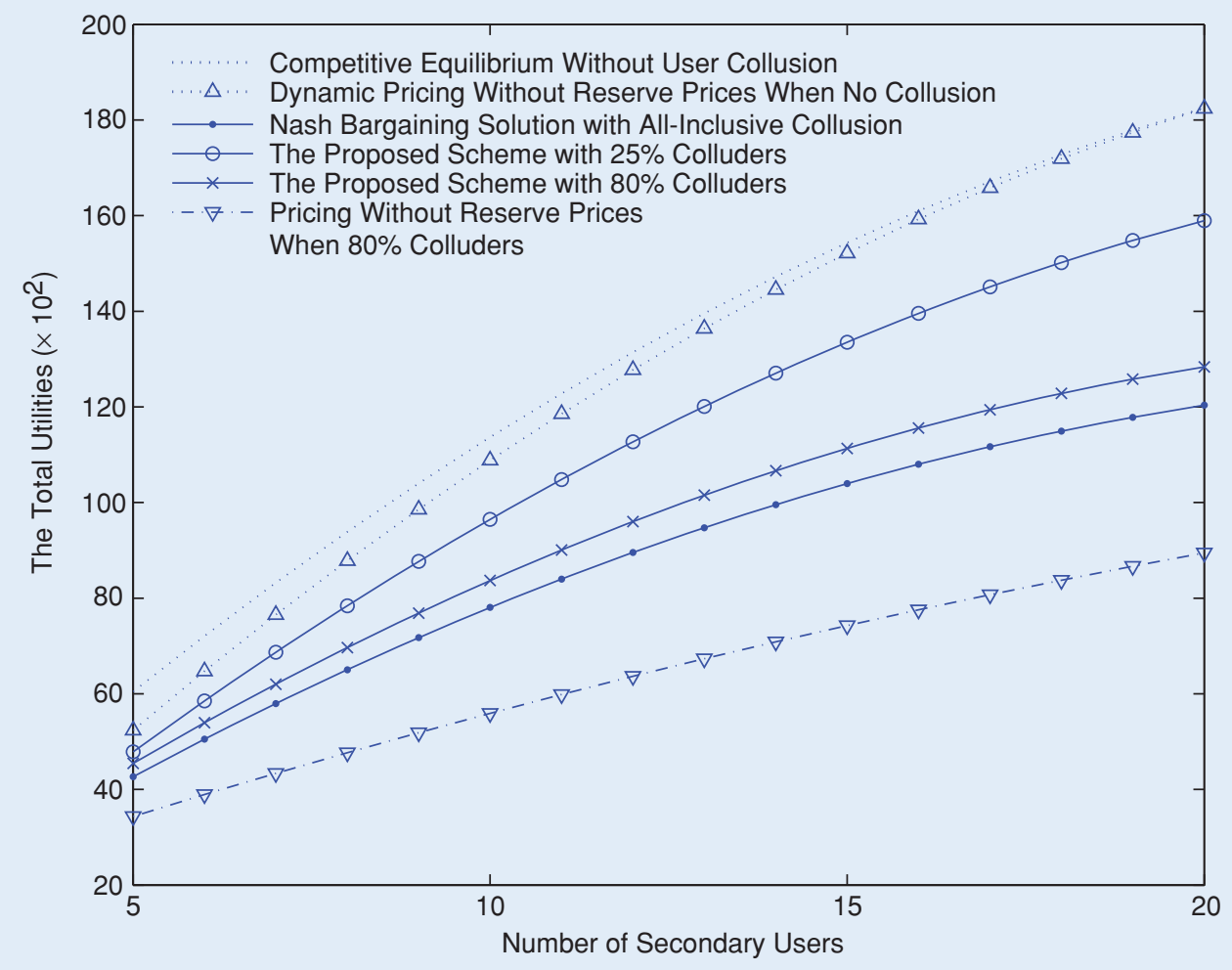

[FIG7] Comparison of the total utilities of the CE, pricing scheme without reserve prices, and the proposed scheme with different user collusion. 
primary users' locations are fixed and their unused channels are available to the secondary users within a distance of $50 \mathrm{~m}$. Then, we randomly deploy $K$ secondary users in the network, which are assumed to be mobile devices. The mobility of the secondary users is modeled using a simplified random waypoint model as in [21]. Let the cost of an available channel in the spectrum pool be uniformly distributed in [10,30], and the reward payoff of leasing one channel be uniformly distributed in [20,40]. If a channel is not available to some secondary users, let the corresponding reward payoffs of this channel be zero. We simulate the case with $J=5$ primary users and 1,000 spectrum sharing stages. Assume each primary user has four unused spectrum channels and the discount factor of the repeated game is 0.99 .

In Figure 7, we compare the total utilities of the optimal competitive equilibrium (CE) [19], our dynamic pricing scheme with reserve prices, and our dynamic pricing scheme without reserve prices under various situations of user collusion. It can be seen that when there is no user collusion, the dynamic pricing scheme without reserve prices is able to achieve similar performance when compared to the theoretical CE outcomes. Moreover, with the presence of user collusion, the proposed scheme with reserves prices achieves much higher total utilities than those of the scheme without reserve prices.

We study the effect of user collusion for dynamic spectrum allocation when each secondary user is constrained by a mone- tary budget [19], in which each secondary user needs to optimally allocate the budget among multiple pricing stages. For comparison, we define a static scheme in which the secondary users make their spectrum-leasing decisions without considering their budget limits. In

Figure 8, we compare the total utilities of our proposed scheme with those of the static scheme for different budget constraints when user collusion is present. It can be seen from the figure that in the presence of user collusion, our proposed scheme with reserve prices achieves significant performance gains over the static scheme when the budget constraints are taken into consideration. That's because the performance loss due to setting reserve prices can be partly offset by exploiting the time diversity of spectrum resources among multiple sharing stages.

\section{CONCLUSIONS}

Dynamic spectrum access in cognitive radio networks has shown its great potential to solve the conflict between limited spectrum resources and increasing demand for wireless services. However, cooperation issues, such as cheating and user collusion, have not been well addressed. In this article, we present two mechanism design based approaches to achieve efficient and cheat-proof spectrum allocation. With the Bayesian mechanism design, the selfish users have no incentive to cheat and can achieve cooperative unlicensed spectrum sharing under the threat of punishment. With collusion-resistant dynamic

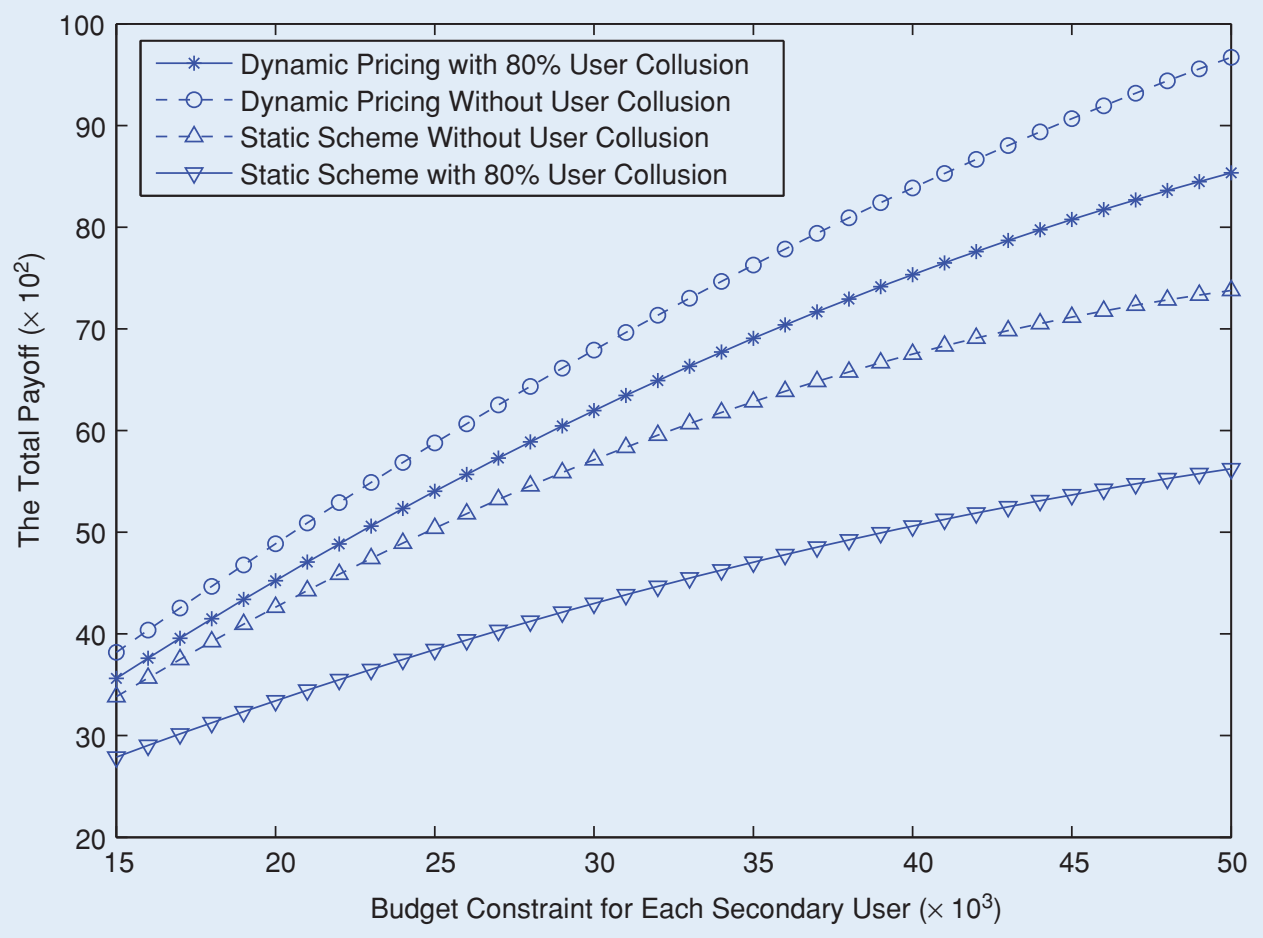

[FIG8] Comparison of the total utilities of the proposed scheme with those of the static scheme. 
spectrum pricing, licensed spectrum resources are efficiently distributed among multiple primary and secondary users, and user collusion is effectively suppressed by setting up the optimal reserve price in the auction.

\section{AUTHORS}

Beibei Wang (bebewang@umd.edu) received her B.S. degree in electrical engineering (with highest honor) from the University of Science and Technology of China, Hefei, in July 2004. She is currently a Ph.D. student in the Department of Electrical and Computer Engineering, University of Maryland, College Park. Her research interests include resource allocation and management in cognitive radio systems, wireless communications and networking, game theory, wireless multimedia, and security. She received the Graduate School Fellowship from the University of Maryland in 2004-2006.

YongleWu (wuyl@umd.edu) received the B.S. and M.S. degrees in electronic engineering from Tsinghua University, Beijing, China, in 2003 and 2006, respectively. He is currently working towards the Ph.D. degree in the Department of Electrical and Computer Engineering, University of Maryland, College Park. His current research interests are in the areas of wireless communications and networks, including cognitive radio techniques, dynamic spectrum access, and network security. He received the Graduate School Fellowship from the University of Maryland in 2006.

ZhuJi (zji@qualcomm.com) received a Ph.D. degree in electrical and computer engineering from the University of Maryland, College Park in 2007 and the B.S. and M.S. degrees in electronic engineering from Tsinghua University, Beijing, China, in 2000 and 2003, respectively. He joined Qualcomm, San Diego, in 2007. From 2003 to 2007, he was a graduate research assistant in the Communication and Signal Processing Laboratory, University of Maryland, College Park. From 2000 to 2002, he was a visiting student (research intern) in the Wireless and Networking Group at Microsoft Research Asia, Beijing, China. His research interests are in wireless communications and networking.

K.J. Ray Liu (kjrliu@umd.edu) is a Distinguished ScholarTeacher of University of Maryland, College Park. He is the recipient of numerous honors and awards including best paper awards from IEEE and EURASIP, IEEE Signal Processing Society Distinguished Lecturer, and National Science Foundation Young Investigator Award. He also received various teaching and research recognitions from University of Maryland. He is vice presidentPublications for the IEEE Signal Processing Society. He was editor-in-chief of IEEE Signal Processing Magazine and founding editor-in-chief of EURASIP Journal on Applied Signal Processing. His recent books include Cooperative Communications and Networking (Cambridge University Press), Resource Allocation for Wireless Networks: Basics, Techniques, and Applications (Cambridge University Press), Ultra-Wideband Communication Systems: The Multiband OFDM Approach (IEEE-Wiley), NetworkAware Security for Group Communications (Springer), and Multimedia Fingerprinting Forensics for Traitor Tracing (Hindawi). He is a Fellow of the IEEE.
T. Charles Clancy (clancy@LTSnet.net) is a senior researcher with the Laboratory for Telecommunications Sciences, and an adjunct professor of electrical engineering at the University of Maryland, College Park. He received his M.S. in electrical engineering from the University of Illinois and his Ph.D. in computer science from the University of Maryland, where his research focused on applications of information theory to cryptography and dynamic spectrum access, respectively. His current research interests involve next-generation wireless networks, specifically software-defined and cognitive radio.

\section{REFERENCES}

[1] J. Mitola III, "Cognitive radio: An integrated agent architecture for software defined radio," Ph.D. dissertation, Dept. Teleinformatics, KTH, Stockholm, Sweden, 2000.

[2] S. Haykin, "Cognitive radio: Brain-empowered wireless communications," IEEE J. Select. Area Commun., vol. 23, no. 2, pp. 201-220, Feb. 2005.

[3] I.F. Akyildiz, W. Lee, M.C. Vuran, and S. Mohanty, "Next generation/dynamic spectrum access/cognitive radio wireless networks: A survey," Comput. Netw. vol. 50, no.13, pp. 2127-2159, Sept. 2006.

[4] Federal Communications Commission, "Facilitating opportunities for flexible, efficient and reliable spectrum use employing cognitive radio technologies: Notice of proposed rule making and order," FCC Document ET Docket No. 03-108, Dec. 2003.

[5] R. Etkin, A. Parekh, and D. Tse, "Spectrum sharing for unlicensed bands," IEEE J. Select. Area Commun., vol. 25, no. 3, pp. 517-528, Apr. 2007.

[6] B. Wang, Z. Ji, and K.J.R. Liu, "Self-learning repeated game framework for distributed primary-prioritized dynamic spectrum access," in Proc. IEEE SECON 2007, San Diego, CA, June 2007, pp. 631-638.

[7] L. Cao and H. Zheng, "Distributed spectrum allocation via local bargaining," in Proc. IEEE SECON 2005, Santa Clara, CA, Sept. 2005, pp. 475-486.

[8] Z. Han, C. Pandana, and K.J.R. Liu, "Distributive opportunistic spectrum access for cognitive radio using correlated equilibrium and no-regret learning," in Proc. IEEE WCNC 2007, Hong Kong, Mar. 2007, pp. 11-15.

[9] J. Huang, R. Berry, and M.L. Honig, "Auction-based spectrum sharing," ACM Mobile Netw. Applicat. (MONET), vol. 11, no. 3, pp. 405-408, June 2006.

[10] S. Gandhi, C. Buragohain, L. Cao, H. Zheng, and S. Suri, "A general framework for wireless spectrum auctions," in Proc. IEEE DySPAN 2007, Dublin, Apr. 2007, pp. 22-33.

[11] O. Ileri, D. Samardzija, and N.B. Mandayam, "Demand responsive pricing and competitive spectrum allocation via a spectrum server," in Proc. IEEE DySPAN 2005, Baltimore, MD, Nov. 2005, pp. 194-202.

[12] D. Niyato and E. Hossain, "Integration of WiMAX and WiFi: Optimal pricing for bandwidth sharing," IEEE Commun. Mag., vol. 45, pp. 140-146, May 2007.

[13] Z. Ji and K.J.R. Liu, "Belief-assisted pricing for dynamic spectrum allocation in wireless networks with selfish users," in Proc. IEEE SECON 2006, Reston, VA, Sept. 2006, pp. 119-127.

[14] Z. Ji and K.J.R. Liu, "Dynamic spectrum sharing: A game theoretical overview," IEEE Commun. Mag., vol. 45, pp. 88-94, May 2007.

[15] L. Hurwicz, "The design of mechanism for resource allocation," Amer. Econ. Rev., vol. 63, no. 2, pp. 1-30, May 1973.

[16] D. Fudenberg and J. Tirole, Game Theory. Cambridge, MA: MIT Press, 1991.

[17] M.J. Osborne and A. Rubinstein, A Course in Game Theory. Cambridge, MA: MIT Press, 1994.

[18] V. Krishna, Auction Theory. New York: Academic, 2002.

[19] Z. Ji and K.J.R. Liu, "Multi-stage pricing game for collusion-resistant dynamic spectrum allocation," IEEE J. Select. Area Commun., vol. 26, no. 1, pp. 182-191, Jan. 2008.

[20] T.A. Weiss, J. Hillenbrand, A. Krohn, and F.K. Jondral, "Efficient signaling of spectral resources in spectrum pooling systems," in Proc. 10th Symp. Communications and Vehicular Technology (SCVT), Nov. 2003 [Online]. Available: htpp://www.teco.edu/ krohn/efficient_signaling.pdf

[21] D.B. Johnson and D.A. Maltz, "Dynamic source routing in ad hoc wireless networks, mobile computing," in Mobile Computing. Norwell, MA: Kluwer, 1996, ch. 5, pp. 153-181.

[22] R.D. Yates, C. Raman, and N.B. Mandayam, "Fair and efficient scheduling of variable rate links via a spectrum server," in Proc. IEEE ICC, Istanbul, Turkey, June 2006, pp. 5246-5251.

[23] A. Papoulis, Probability, Random Variables, and Stochastic Processes, 3rd ed. New York: McGraw-Hill, 1995. 